\title{
An Experimental Study of Team Effectiveness and Satisfaction in an Engi- neering Design Course
}

\section{Dr. Nausheen PashaZaidi, The Petroleum Institute}

Dr. Nausheen Pasha-Zaidi is an Assistant Professor in the General Studies Department at the Petroleum Institute in Abu Dhabi. Her research interests focus on issues in international psychology and education with an emphasis on perceptions, attitudes, and stereotypes in intercultural communication. Her novel, The Colour of Mehndi, is a fictionalized biopic that explores the experience of immigration and acculturation among first generation Pakistani-Americans.

\section{Dr. Jaby Mohammed, The Petroleum Institute}

Jaby Mohammed is a faculty at The Petroleum Institute, Abu Dhabi, UAE. He received his PhD in Industrial Engineering from University of Louisville (2006), masters in Industrial Engineering from University of Louisville (2003) and also a master's in business administration from Indira Gandhi National Open University (2001). His research interests include advanced manufacturing, design methodologies, six sigma, lean manufacturing, and engineering education. He previously taught at Indiana Purdue Fort Wayne, IN and at Morehead State University, KY. He is a member of IIE, SME, ASQ, ASEE, and Informs. 


\title{
An Experimental Study of Team Effectiveness and Satisfaction in an Engineering Design Course
}

\begin{abstract}
Many countries are experiencing a shortfall of trained engineers and are working to recruit and retain their own citizens to study in science, technology, engineering and mathematics (STEM) fields to build their intellectual capital. The United Arab Emirates is one such example. The Petroleum Institute (PI) provides opportunities for students to grow as engineering professionals and make positive contributions to future employers.

Perceptions of effective group dynamics and the contributions of individual team members to the group process can affect the quality of the team experience. In this study, female students in an Engineering Design course at the PI were randomly assigned to teams. After the first team presentation, they were asked to consider the effectiveness of their random team assignment and reflect on their team processes. Student perceptions of team effectiveness were gauged using anonymous surveys. Engagement in teamwork was subsequently assessed using a variety of tools. In this paper, the authors discuss the implications of choice in team creation on student perceptions of team effectiveness and satisfaction.
\end{abstract}

\section{Introduction}

Research suggests that same-gender teams (all-male or all-female) perceive themselves as more effective than heterogeneous teams (Baugh \& Graen, 1997). In classroom situations, it appears that both genders value the importance of teamwork, but more female students want to be able to choose their own team members (Alexander \& Stone, 1997). As task interdependence is a factor in effective teamwork (Ramamoorthy \& Flood, 2004), it is possible that homogenous teams offer a greater degree of comfort for communication among team members to address the tasks needed. For female students, control over team memberships may be linked to a higher degree of comfort and familiarity with members' abilities and skills. In Arab communities in the Middle East, gender homogeneity and communication patterns among gender-segregated groups may enhance this phenomenon. The current study was undertaken with a cohort of all-female students at the Petroleum Institute (PI) in Abu Dhabi to explore the effect of student choice in team creation on their team effectiveness and satisfaction.

\section{Background}

The mission of the PI is to impart high quality engineering education and research in order to support and advance the energy industries. The Institute strives to develop students as future leaders in their respective fields of expertise in the UAE and globally by placing a focus on the development of professional skills that students can apply to the workplace. Within this context, a primary goal is the ability to cultivate teamwork skills in engineering practice and project management. The General Studies department at the 
PI offers a sequence of two design courses known as STEPS (Strategies for Team-based Engineering Problem Solving) which stress team learning and skills development. In STEPS courses students integrate their knowledge of science, mathematics and communications within a framework that emphasizes teamwork and project management tools to build a working prototype of a useful machine.

\section{Communication and Teamwork in the UAE}

Student demographics at the PI reflect an Arab majority with Emirati students playing a key role. Arab communities display some common features which may differentiate them from their Western counterparts. This includes an emphasis on collectivism, honor, and social status within extended family and tribal relations (Feghali, 1997; Al-Krenawi, \& Graham, 2000). Some of the general communication patterns that appear in Arab communities include code-switching from one language to another, direct speech when commenting on personal appearances, but indirect speech when discussing general social practices or norms. Physical space tends to be closer in all-male or all-female situations as public displays of contact and touching between genders is considered offensive. Status resulting from age or tribal affiliation may also influence communication patterns (Feghali, 1997). The local Emiratis are highly embedded in tradition. Within the Emirati culture, communication patterns may reflect more traditional roles and expectations than communication between Emiratis and expatriates.

The quality of team processes and team performance may be influenced by demographic characteristics and patterns of social interaction (Rentsch \& Hall, 1994). Rogelberg \& Rumery (1996), for example, found that gender homogeneity increased team performance for females. In a study of homogeneity of subgroups, Gibson \& Vermeulen (2003) found that homogeneous subgroups were better able to experiment and implement solutions. Additionally, a positive correlation was noted between communication and team effectiveness among employees in Abu Dhabi government departments (Al-Romaithi, 2011). It is possible, then, that single-gender homogenous teams who share a cultural schema may have better communication among team members. On the other hand, homogeneity of gender and culture may also encourage groupthink which would perhaps have a negative influence on creativity in the group. In a study by Bennet and Wright (2010), female Emirati students in a single-gender, single-nationality university reported having more positive experiences than Emirati female students in a mixed-gender university. However, they were also more likely to hide their own opinions and change their views based on the opinions of the group.

\section{Participants}

The present study was conducted with twenty female students in an engineering design course (STEPS 201) at the Petroleum Institute in Abu Dhabi. The class consisted of freshmen and sophomores on the "Arzanah" campus, which is gender segregated for female students. Student ages ranged from 18-20 $(n=20)$. The majority of students were Emirati nationals $(n=18)$ and all students were of Arab descent. 


\section{Rationale}

The "Strategies for Team Based Engineering Problem Solving" (STEPS) Program is composed of two courses focusing on engineering design. The overall goal of the STEPS program is to introduce students to the engineering design process and integrate a range of professional skills and competencies that will simulate real-world design activities, such project management, reverse engineering, and computer aided design. This program requires teams of students to respond to client specifications by designing, managing and presenting technically feasible solutions to real-world problems. Teamwork, organization, planning, research skills, and problem solving are essential for success. All students are actively engaged in teamwork and solving open-ended problems using methodical approaches and state-of-the-art design and communications tools. During the semester, students also regularly present the results of their project work using oral, written and graphical communication skills. The progress and development of each team project and each student's contribution is closely monitored and evaluated by qualified engineering design experts and qualified English communication specialists for the purpose of providing helpful feedback and advice to improve project work and team work skills. The course focuses on team processes and so, half of the assignments are assessed with team grades. Tasks require interdependency and time management as lectures are kept to a minimum and students are encouraged to utilize their time efficiently, both during and after class hours, for designing their team project. In general, team members are chosen randomly by instructors and team membership is not negotiable or transferrable during the semester.

The current study was initiated based on reports of discontentment in team assignments communicated by the students to the instructors during the first few weeks after team selection and after their first team presentations. At that point, teams had been working together for about 5 weeks and had created a team charter representing their team goals as well as team member strengths and weaknesses. A survey was utilized to gain anonymous feedback regarding students' perceptions of their team assignments. Initial survey results showed that the majority $(68.4 \%)$ of students in the course preferred to change their group memberships, despite the fact that they felt that their current group was effective in terms of task performance. The majority $(73.7 \%)$ also reported that the best way to create teams is to allow students to choose their own team members. This supports the findings of Alexander and Stone (1997) with regard to female teams. Based on these results, the instructors allowed students to assign themselves to their own groups using a sign-up sheet that was distributed during class. Students were free to remain in their current group or to choose new group members. Results of the sign-up sheet indicated that all students had chosen different team members.

Students were given one day to reflect on their new teams and begin work on new team charters. Despite the fact that all students in the class chose their group members, there were still echoes of discontentment as individual students visited the instructors to provide their criticism on the team changes. In order to address the issue and ensure that the majority was satisfied with the change, another anonymous survey was used the following day to get additional feedback. When asked if students wanted to continue with 
their new team or return to their original team assignments, the majority of students chose to return to their original teams. Reasons for returning to the original team assignments included a sense of commitment to the original team members, consistency of established communication protocols with the original team members, and a preference for continuity with the original team.

As teams began work on the new project, they were required to post weekly team minutes illustrating their group activities. Updates on teamwork were also provided through weekly meetings with the instructors. Finally, a survey utilizing both Likert-style questions and open-ended responses was administered after the design project presentation towards the end of the semester to allow students to individually reflect on their team processes.

\section{Results and Discussion}

The present study followed a pre-test/post-test format to determine student attitudes towards group creation, team effectiveness, and team satisfaction. The pre-test condition consisted of the survey administered to students to assess their initial attitudes prior to being given the choice to change group members. The post-test condition used a similar survey at the end of the semester to determine what, if any, changes had occurred in terms of student attitudes towards group creation, team effectiveness and satisfaction.

Regarding group creation, results from the pre-test condition indicated that $73.7 \%$ of the students felt that they should have the opportunity to select their own group members. Results from the post-test indicated that $63.2 \%$ of the students wanted to select their own group members (See Figure 1 and 2 below).

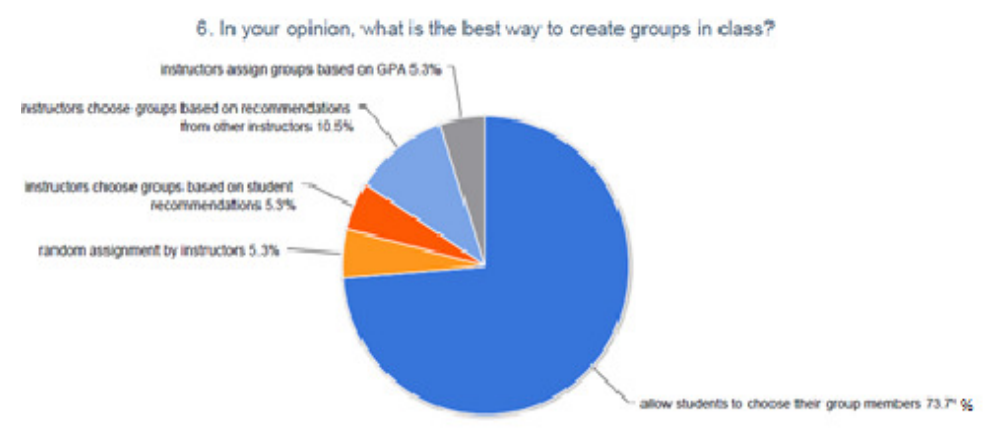

Figure 1: group creation - pre-test condition 


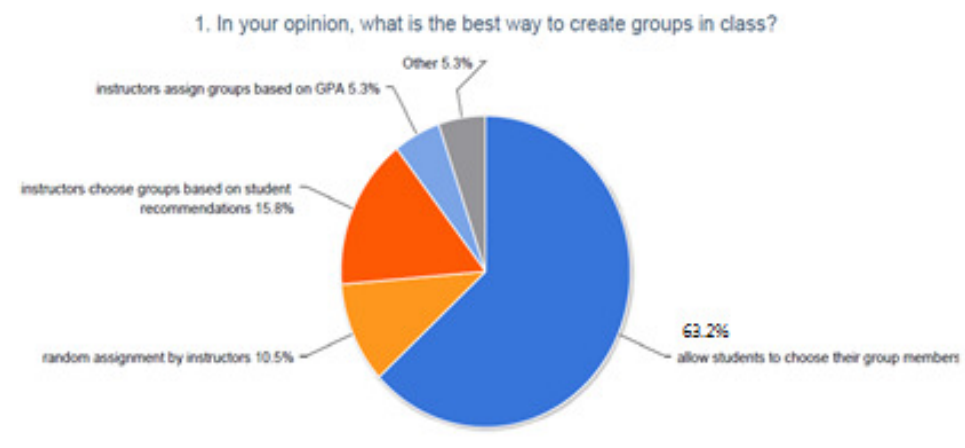

Fig 2: group creation - post-test condition

The change in attitude towards group creation at the end of the semester may have been the result of positive interactions among the group members over time, which may have lessened some students' desire to have control over the choice of group members. Additionally, remarks made by students at the time of the experimental condition (when the majority decided to return to the original randomly-assigned team) indicated that the teams had begun to jell even then. Some of these comments included the following: "I think that the old teams didn't cause any problems and they should be put together again"; Both groups are fine but I prefer the previous group because I can communicate easily with them..."; "I already had a chance to work with the old group"; and "I felt bad about leaving my old group because I was very comfortable working with them and as for the new team I would be having more load than my friends (experienced working with them before)". Thus, it is possible that students who are given the choice to create their own teams may feel pressured to choose their friends, but if the choice is taken away, the peer pressure involved may be lessened. The following comments speak to this: "Some of the girls were not satisfied with their new group, they thought that it was not fair, therefore I thought that my previous group is as good as my new one, so why not"; Sometimes being in a group with friends, we tend to be lazy and if one of us had to go home or has other things, we will say okay. The work will be delayed".

With regards to team effectiveness, in the pre-test condition, $89.5 \%$ of students reported that their team was either somewhat effective (15.8\%), moderately effective $(63.2 \%)$ or highly effective $(10.5 \%)$. Another $10.6 \%$ felt that their group was either ineffective or highly ineffective. In the post-test condition, $100 \%$ of the students reported that their team was within the effective category with $31.6 \%$ reporting that their group was highly effective (Table 1). This may once again support the notion of allowing students time to jell as a team and get to know each other's personalities and work habits. Thus, early in the team-building process, students may be more focused on the individuals that make up the team, but as the teamwork progresses, the team's energy may focus more on the tasks at hand, allowing the team to become more effective over time. The mean grade after the first team project for the class was 87.3 and the mean grade for the final poster presentation was 89.8 . Thus, not only their perceptions of effectiveness improved over the semester, but so did their team effectiveness as evidenced by their team grades. 
Table 1: Team Effectiveness (Pre-test and Post-test)

\begin{tabular}{|ll|lc|}
\hline Pretest Condition: & & Post-test Condition: & \\
& & \\
Team Effectiveness & Percent & Team Effectiveness & Percent \\
Highly Ineffective & $5.3 \%$ & Highly ineffective & $0.0 \%$ \\
Ineffective & $5.3 \%$ & Ineffective & $0.0 \%$ \\
Somewhat Effective & $15.8 \%$ & Somewhat effective & $15.8 \%$ \\
Moderately Effective & $63.2 \%$ & Moderately effective & $52.6 \%$ \\
Highly Effective & $10.5 \%$ & Highly effective & $31.6 \%$ \\
& & & \\
\hline
\end{tabular}

With regards to team satisfaction, in the pre-test condition, $47.4 \%$ reported being neutral, another $47.4 \%$ reported being satisfied with their current team, and 5.3\% reported being dissatisfied with their team. At the end of the semester, there was a greater range of responses; $10.5 \%$ indicated that they were highly satisfied with their team, $47.4 \%$ reported being satisfied, $26.3 \%$ remained neutral and $15.8 \%$ were dissatisfied (Table 2 below). As the experimental condition of allowing students to change their team members took place after their first team presentation, it is possible that this caused students to assess their satisfaction level in a different manner at the end of the semester. As there was not $100 \%$ agreement to changing the teams back to the original randomized team allocations, some students remained dissatisfied with their teams. The changes in team allocations may have created additional stress among the students, which may have affected their satisfaction level.

Table 2: Team Satisfaction (Pre-test and Post-test)

\begin{tabular}{|ll|ll|}
\hline Pre-test Condition: & & Post-test Condition: & \\
& & \\
Team Satisfaction & Percent & Team Satisfaction & Percent \\
Very Dissatisfied & $0.0 \%$ & Very Dissatisfied & $0.0 \%$ \\
Dissatisfied & $5.3 \%$ & Dissatisfied & $15.8 \%$ \\
Neutral & $47.4 \%$ & Neutral & $26.3 \%$ \\
Satisfied & $47.4 \%$ & Satisfied & $47.4 \%$ \\
Very Satisfied & $0.0 \%$ & Very Satisfied & $10.5 \%$ \\
& & & \\
\hline
\end{tabular}

\section{Recommendations for Effective Teamwork in Homogenous Groups}

The empowerment of team members has been shown to be a factor in effective teamwork to stimulate learning-related activities (Cohen \& Ledford, 1994; Kirkman \& Shapiro, 1997; Hyatt \& Ruddy, 1997; Kirkman \& Rosen, 1999). The present study hoped to provide some level of empowerment to the students in terms of choosing their own team members. However, as the experimental condition was implemented after students had 
started teamwork with instructor-assigned teams, the opportunity for empowerment in team creation may have created more stress in the process. Thus, it is important to bear in mind that empowerment of teams does not necessarily mean choice in team member selection. The following recommendations may be useful:

- Give teams time to understand each other and form a team identity (Katz, 2001). Despite the fact that all students in the current study were Arab females and the majority of teams consisted of all Emirati females, complaints about team membership were brought up during the early stages of the random team formation. As teams worked together over the semester, however, they became familiar with each other's habits and skills, allowing the focus to shift from individual team members to task completion. Thus, it is important to remember that intra-group variation and sub-group preferences can play a role in group processes, even within same-gender teams that appear to be culturally homogenous. Dissolving teams in reaction to student complaints in the early stages of team development may be less effective than allowing students to work out their differences over time.

- When creating randomized teams, allow students some level of choice, such as getting recommendations for team membership or allowing students to choose a partner on the team. A subgroup within the larger group can be useful for students because it provides them with the psychological support of at least one group member with whom they are familiar. Having a "friend" on the team may lessen the fear of embarrassment so that students can voice their opinions with the knowledge that at least one team member is more likely to stand by them (Gibson \& Vermeulen, 2003)

- Have students create a team charter and use it throughout the project. Effective teams within the classroom environment should model professional contexts and a team charter is one way of providing a framework for acceptable behavior. Cox, College, and Bobrowski (2000) note that having a shared vision and clear team goals can enhance productivity among team members. Additionally, setting team expectations at the outset can deter social- loafing behaviors (Katzenbach and Smith, 1993). A team charter can be a useful tool (Cox, College, \& Bobrowski, 2000; Hunsaker, Pavett, \& Hunsaker, 2011), but only if it is actually implemented during team processes. 


\section{References}

Alexander, M. W., \& Stone, S. F. (1997). Student Perceptions of Teamwork in the Classroom: An Analysis by Gender. In Business Education Forum (Vol. 51, No. 3, pp. 7$10)$.

Al-Khazraji, N. (2009). The culture of commercialism: globalization in the UAE (Doctoral dissertation, Georgetown University Washington, DC).

Al-Krenawi, A., \& Graham, J. R. (2000). Culturally sensitive social work practice with Arab clients in mental health settings. Health \& Social Work, 25(1), 9-22.

Al Romaithi, A. A. (2011). Organisational Culture and Teamwork in Governmental Organization: The Case of the UAE (Doctoral dissertation, British University in Dubai).

Baugh, S. G., \& Graen, G. B. (1997). Effects of team gender and racial composition on perceptions of team performance in cross-functional teams. Group \& Organization Management, 22(3), 366-383.

Cohen, S. G., \& Ledford, G. E. (1994). The effectiveness of self-managing work teams: A quasi-experiment. Human Relations, 47, 643-676.

Cox, P. L., \& Bobrowski, P. E. (2000). The team charter assignment: Improving the effectiveness of classroom teams. Journal of Behavioral and Applied Management, 1(1), 92-103.

Feghali, E. (1997). Arab cultural communication patterns. International Journal of Intercultural Relations, 21(3), 345-378.

Hunsaker, P., Pavett, C., \& Hunsaker, J. (2011). Increasing student-learning team effectiveness with team charters. Journal Of Education For Business, 86(3), 127-139. doi:10.1080/08832323.2010.489588

Hyatt, D. E., \& Ruddy, T. M. (1997). An examination of the relationship between work group characteristics and performance: Once more into the breech. Personnel Psychology, 50(3), 553-585.

Katz, N. (2001). Sports teams as a model for workplace teams: lessons and liabilities. The Academy of Management Executive, 15(3), 56-67.

Katzenbach, J. R., \& Smith, D. K. (1993). The discipline of teams (pp. 111-120). Harvard Business Press.

Kirkman, B. L., \& Rosen, B. (1999). Beyond self-management: Antecedents and consequences of team empowerment. academy of Management Journal, 42(1), 58-74. 
Kirkman, B. L., \& Shapiro, D. L. (1997). The impact of cultural values on employee resistance to teams: Toward a model of globalized self-managing work team effectiveness. Academy of Management Review, 22(3), 730-757.

Ramamoorthy, N., \& Flood, P. C. (2004). Individualism/collectivism, perceived task interdependence and teamwork attitudes among Irish blue-collar employees: a test of the main and moderating effects?. Human Relations, 57(3), 347-366.

Rentsch, J. R., \& Hall, R. J. (1994). Members of great teams think alike: A model of team effectiveness and schema similarity among team members.

Rogelberg, S. G., \& Rumery, S. M. (1996). Gender diversity, team decision quality, time on task, and interpersonal cohesion. Small Group Research, 27(1), 79-90. 